\title{
Electronic Structure-Dependent Surface Plasmon Resonance in Single Au-Fe Nanoalloys
}

Duncan T. L. Alexander, ${ }^{*, \dagger, \ddagger \odot}$ Daniel Forrer, ${ }^{\|,}$Enrico Rossi, ${ }^{\S}$ Elefterios Lidorikis, ${ }^{\perp}{ }^{\circledR}$ Stefano Agnoli, ${ }^{\S}$

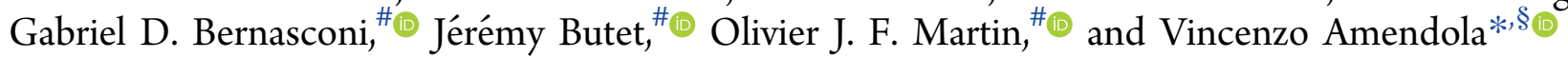

${ }^{\dagger}$ Electron Spectrometry and Microscopy Laboratory (LSME), Institute of Physics (IPHYS), Ecole Polytechnique Fédérale de Lausanne (EPFL), 1015 Lausanne, Switzerland

${ }^{\ddagger}$ Interdisciplinary Centre for Electron Microscopy (CIME), Ecole Polytechnique Fédérale de Lausanne (EPFL), 1015 Lausanne, Switzerland

"CNR-ICMATE, 35127 Padova, Italy

${ }^{\S}$ Department of Chemical Sciences, University of Padova, 35131 Padova, Italy

${ }^{\perp}$ Department Materials Science and Engineering, University of Ioannina, 45110 Ioannina, Greece

${ }^{\#}$ Nanophotonics and Metrology Laboratory (NAM), Ecole Polytechnique Fédérale de Lausanne (EPFL), 1015 Lausanne, Switzerland

\section{Supporting Information}

ABSTRACT: The relationship between composition and plasmonic properties in noble metal nanoalloys is still largely unexplored. Yet, nanoalloys of noble metals, such as gold, with transition elements, such as iron, have unique properties and a number of potential applications, ranging from nanomedicine to magneto-plasmonics and plasmon-enhanced catalysis. Here, we investigate the localized surface plasmon resonance at the level of the single $\mathrm{Au}-\mathrm{Fe}$ nanoparticle by applying a strategy that combines experimental measurements using near field electron energy loss spectroscopy with theoretical studies via a full wave numerical analysis and density functional theory calculations of electronic structure. We show that, as the iron fraction increases, the plasmon resonance is blue-shifted and significantly damped, as a consequence of the changes in the electronic band structure of the alloy. This allows the identification of three relevant phenomena to be considered in the design and realization of any plasmonic nanoalloy, specifically: the appearance of new states around the Fermi level; the change in the free electron density of the metal; and the blue shift of interband transitions. Overall, this study provides new opportunities for the control of the optical response in $\mathrm{Au}-\mathrm{Fe}$ and other plasmonic nanoalloys, which are useful for the realization of magnetoplasmonic devices for molecular sensing, thermo-plasmonics, bioimaging, photocatalysis, and the amplification of spectroscopic signals by local field enhancement.

KEYWORDS: plasmon resonance, alloy, nanoparticles, EELS
A wealth of applications are based on the control of lightmatter interaction in metal nanoparticles (NPs) through physical processes related to plasmons, namely, the collective oscillations of conduction electrons in metallic systems. ${ }^{1}$ Indeed, the localized surface plasmon resonance (LSPR) in metal NPs can be tailored in order to achieve strong near-field electromagnetic enhancement effects, specific far field scattering properties, intense light extinction, efficient photothermal transduction, or even hot-carriers generation. ${ }^{2-6}$ Exploiting such versatility for target applications requires great efforts in the development of advanced fabrication methods for plasmonic NPs, in order to completely control the nanostructures' size, geometry, assembly, and dielectric environment. $^{6,7}$ Another parameter for tuning the LSPR response and, in particular, for obtaining new physicalchemical properties is the alloying of traditional plasmonic materials such as gold and silver with other elements from the periodic table. ${ }^{8-10}$ The field of plasmonic nanoalloys made of noble metals and transition metals is especially sought for the coexistence of plasmonics with magnetism or catalytic activity. ${ }^{11-13}$ In the best cases, the generation of mutually influenced, physical-chemical phenomena like magnetoplasmonics $^{14,15}$ and plasmon enhanced catalysis ${ }^{16-18}$ are also

Received: June 13, 2019

Revised: July 23, 2019

Published: July 26, 2019 

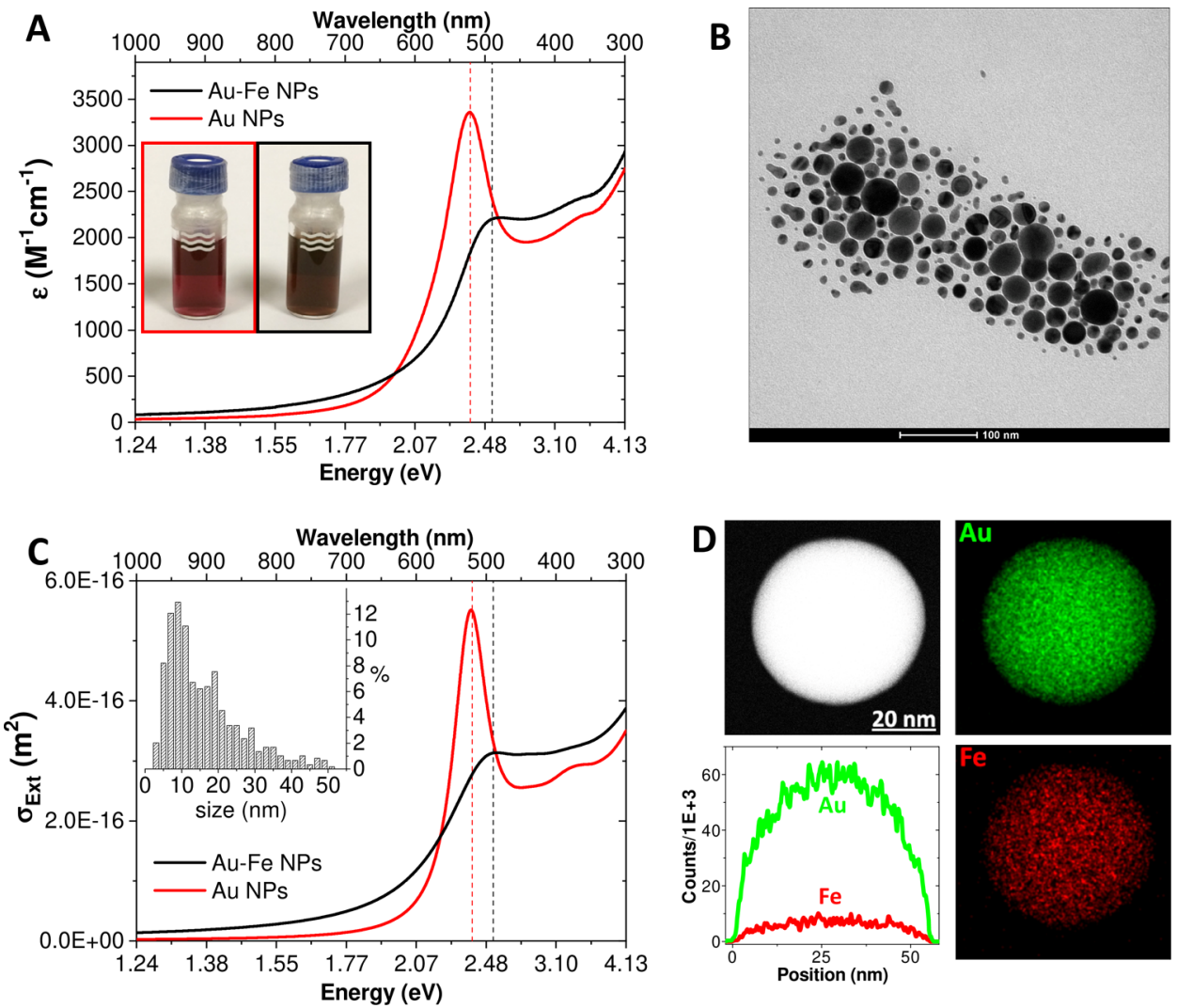

Figure 1. (A) Molar extinction coefficient ( $\varepsilon$, referred to moles of metal atoms) of Au-Fe nanoalloys in water (black line) and of a reference sample of pure Au NPs (red line). The difference in the optical extinction of the two colloids can also be appreciated by the naked eye, as shown in the inset. (B) TEM image of the Au-Fe nanoalloys. (C) Mie model calculations of the extinction cross section $\left(\sigma_{\mathrm{Ext}}\right)$ of $\mathrm{Au}(88) \mathrm{Fe}(12)$ alloy NPs (black line) and of pure Au NPs (red line), providing as input the size distribution measured by TEM on the Au-Fe nanoalloy sample, which is shown in the inset. (D) Elemental analysis of an $\mathrm{Au}-\mathrm{Fe}$ NP containing 85 at \% of Au and 15 at \% of Fe, showing STEM HAADF image (top left); 2D EDXS maps of $\mathrm{Au}(\mathrm{L}-\alpha, \beta$ peaks) and $\mathrm{Fe}$ (K- $\alpha, \beta$ peaks) distribution (right); profile of a linescan across the NP (integrated over a $9.6 \mathrm{~nm}$ width) of the net $\mathrm{Au}$ and $\mathrm{Fe}$ counts for the mapped X-ray peaks (bottom left). The maps and the linescan confirm that the two elements are homogeneously distributed within the NP.

possible. Recent reports have highlighted the unique properties and the multiple applications of plasmonic nanoalloys such as $\mathrm{Au}-\mathrm{Fe}$ and $\mathrm{Ag}-\mathrm{Fe} \mathrm{NPs}$, which were proposed as multimodal contrast agents in nanomedicine, ${ }^{19}$ as magnetically responsive plasmonic substrates in surface enhanced Raman scattering, $^{20,21}$ as efficient converters of light into heat, ${ }^{21,22}$ as well as for use in catalysis. ${ }^{23}$

While the spectroscopic properties of plasmonic nanostructures composed of the same material have been thoroughly studied, both in ensembles and at the single particle level, ${ }^{24}$ the response of multifunctional plasmonic nanoalloys remains largely unexplored. ${ }^{25}$ In order to elucidate the effect of alloying on plasmonic response, a thorough approach is necessary, going from the precise identification of alloy composition and plasmonic response at the single nanoparticle level, to electromagnetic theory and calculations of the electronic structure of the alloy. Experimentally, the alloying between noble metals and base metals is hindered by thermodynamic constraints, such that several attempts to fabricate nanoalloys resulted in heterostructures or core-shell structures. ${ }^{26}$ For instance, a large miscibility gap exists in the $\mathrm{Au}-\mathrm{Fe}$ binary phase diagram, due to the structural dissimilarity of the bodycentered cubic (BCC) iron and face-centered cubic (FCC) gold end members and the large difference in their lattice parameters, with the solubility of $\mathrm{Fe}$ in $\mathrm{Au}$ being estimated to be $<2$ at $\%$ at $200{ }^{\circ} \mathrm{C}^{27}$ Consequently, it was shown that $\mathrm{Au}-$
Fe alloy can be obtained in a wide range of compositions only by out of equilibrium synthetic methods. ${ }^{28}$ To overcome this limitation, a fabrication method was developed that enables the synthesis of iron-doped $\mathrm{Au}$ and $\mathrm{Ag}$ nanoparticles in one step, by a simple and environmentally friendly approach based on laser ablation in liquid. ${ }^{21,29-32}$ Using laser ablation in liquid, metastable $\mathrm{Au}-\mathrm{Fe}$ nanoalloys can be obtained by fast quenching of a high temperature solid solution of the two metals ejected from a bulk target absorbing the laser pulses. ${ }^{27}$ In these nanoalloys, the plasmonic response of the gold moiety and the magnetism of the iron moiety coexist, though with strong modification compared to single element NPs, for instance revealing a nonlinear surface plasmon resonance dependence on the iron fraction, and a transition from paramagnetic to a spin-glass state at low temperature. ${ }^{29}$

Although the far and near field spectroscopic properties of the $\mathrm{Au}-\mathrm{Fe}$ nanoalloys have been investigated in ensembles of nanoparticles, ${ }^{30}$ with an appreciable agreement between experiments and theoretical calculations, to date the plasmonic response at the single nanoparticle level was not probed. This is relevant because, while the average elemental composition of laser generated $\mathrm{Au}-\mathrm{Fe}$ nanoalloys is easily and clearly identifiable, ${ }^{29,30}$ at the single NP level it spans a statistical distribution peaked on the average value, with extremes going from iron-poor to iron-rich conditions. ${ }^{29}$ Also, in a few limited cases, phase segregated heterostructures or core-shell 

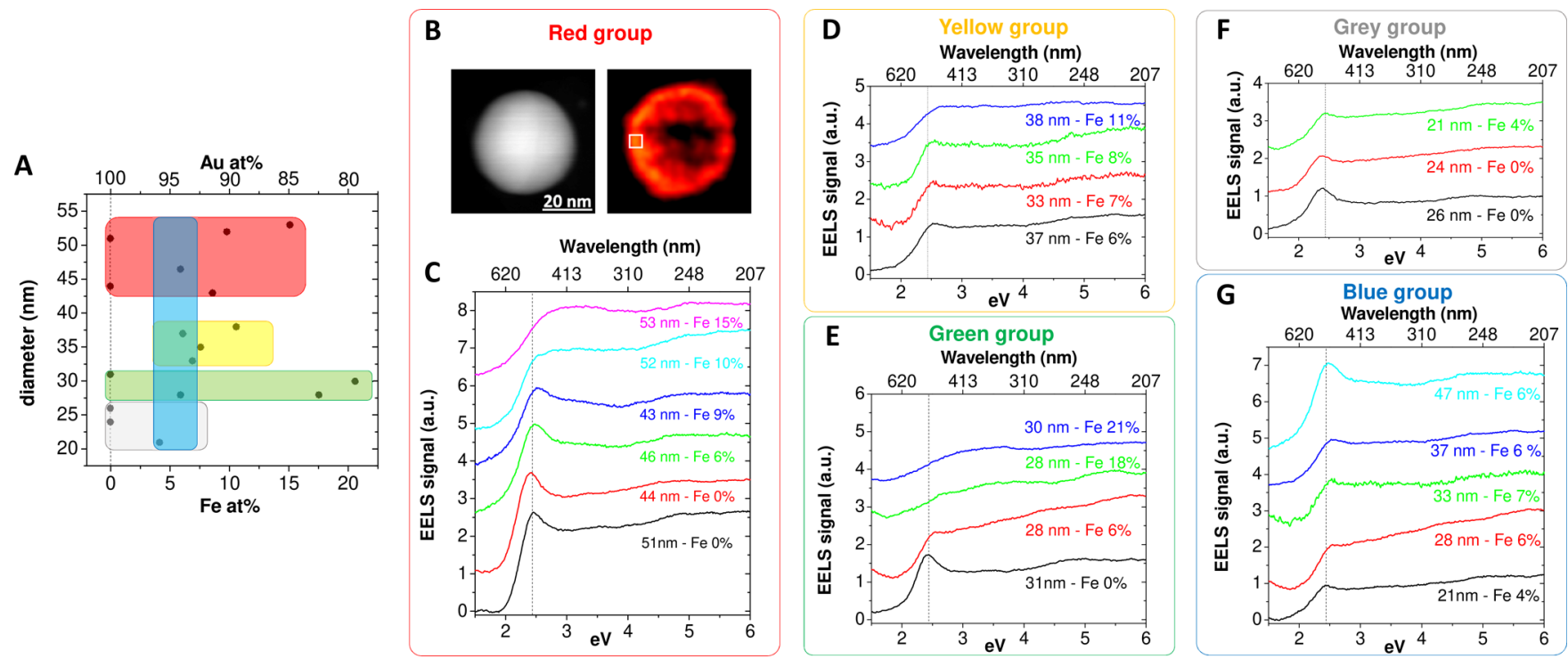

Figure 2. (A) Size versus composition of the $\mathrm{Au}-\mathrm{Fe}$ and $\mathrm{Au}$ NPs analyzed by EELS. (B) STEM HAADF image and representative $2 \mathrm{D}$ EELS map collected at $2.5 \mathrm{eV}$ on a $\mathrm{Au}(91) \mathrm{Fe}(9) \mathrm{NP}$ (43 nm in size), showing the intensity distribution of its dipolar plasmon mode. The white square represents the type of analysis area that was used for acquiring the single EELS spectra reported below. The EELS spectra of all the Au-Fe and Au NPs, grouped in four sets with different composition but similar sizes corresponding to $48 \pm 4,36 \pm 2,29 \pm 2$, and $24 \pm 2 \mathrm{~nm}$, are reported in, respectively, the red (C), yellow (D), green (E), and gray (F) rectangles (same as the colors of the rectangles in (A)). (G) EELS spectra for a set of $\mathrm{Au}-\mathrm{Fe}$ NPs with similar composition of $5.8 \pm 1.0$ at \% and size ranging from 21.0 to $46.5 \mathrm{~nm}$ (identified by the blue rectangle in (A)). The spectra are vertically shifted for an easier comparison.

structures are found together with a majority of homogeneous alloy NPs. ${ }^{31}$

In order to address these limitations, in this Letter we investigate the LSPR at the level of the single $\mathrm{Au}-\mathrm{Fe}$ nanoalloy by near field measurements performed with electron energy loss spectroscopy (EELS) combined with a full wave numerical analysis based on the surface integral equations (SIE) method $^{33,34}$ and density functional theory (DFT) calculations. EELS is based on the measurement of the energy lost by fast electrons owing to their interaction with the sample; such energy loss can be induced via the excitation of surface plasmons when the trajectory of electrons passes close to metallic nanostructures. ${ }^{35,36}$ EELS has been applied to study a broad variety of plasmonic systems, ${ }^{37-44}$ and it has also been used to probe LSPR kinetics and damping in single plasmonic nanostructures. ${ }^{45}$ EELS not only provides information on the near field distributions and the modal nature of the observed peaks, but also an insight into the evolution of the absorption and scattering mechanisms. ${ }^{36,46}$ Indeed, the relative weight of these two loss channels is expected to evolve upon compositional changes, for instance, when going from the well-known case of pure $\mathrm{Au}$ nanoparticles to the unexplored case of $\mathrm{Au}-\mathrm{Fe}$ nanostructures of the same geometry. In addition, in the same instrument, scanning transmission electron microscopy (STEM) energy dispersive X-ray spectroscopy (EDXS) is applied to determine the composition of each analyzed nanoparticle.

Using these methods, here we provide direct insights into the plasmonics of $\mathrm{Au}-\mathrm{Fe}$ nanoalloys, identifying the influence of the NP composition on the electromagnetic response. By relating the plasmon resonances and plasmon-interband interaction with the composition, this work facilitates the development of multielement plasmonic structures with tailored responses, beyond the possibilities offered by singleelement plasmonic ones. Due to the multiple functionalities of the magneto-plasmonic systems, ${ }^{4-53}$ we expect that these results will reveal new opportunities for the control of the nanoscale optical response, as required for efficient tuning of directional scattering, ${ }^{54}$ molecular sensing, ${ }^{55,56}$ photocatalysis, $^{57}$ and local field enhancement for the amplification of spectroscopic signals. ${ }^{34}$

$\mathrm{Au}-\mathrm{Fe}$ nanoalloy solution, in the form of a colloidal dispersion in water, was obtained by the laser ablation approach (see Supporting Information (SI) for details). The crystalline structure of the NPs was characterized using X-ray diffraction (XRD). The XRD pattern (Figure S1 in SI) shows all the peaks typical of the FCC lattice of $\mathrm{Au}$, without other contributions, but for a smaller lattice parameter of $4.031 \AA$ compared to that of pure $\mathrm{Au}(4.079 \AA)$. This is consistent with the formation of a metastable $\mathrm{Au}-\mathrm{Fe}$ alloy, with the $\mathrm{Fe}$ atoms randomly substituting $\mathrm{Au}$ atoms, at the Au lattice sites. ${ }^{23,29,30}$ Further confirmation of this alloy nature is provided by measuring the binding energies of $\mathrm{Fe} 2 \mathrm{p}$ and $\mathrm{Au} 4 \mathrm{f}$ core levels with X-ray photoelectron spectroscopy (XPS), where the Fe component is confirmed as being metallic and the peak positions are consistent with alloy formation (Figure S2 in SI).

Before analyzing the optical properties of the $\mathrm{Au}-\mathrm{Fe}$ nanoalloys on an individual particle basis, we first discuss the optical properties of the ensemble. Their UV-vis absorption spectrum is characterized by a weak plasmon band at $2.53 \mathrm{eV}$ $(490 \mathrm{~nm})$, superimposed on a wide interband absorption edge (black line in Figure 1A). In comparison, for a colloid of pure $\mathrm{Au}$ with similar average particle size of $20 \pm 6 \mathrm{~nm}$ and lognormal size distribution, the intensity of the LSPR is much higher than the interband transition edge and located at 2.38 $\mathrm{eV}(520 \mathrm{~nm}$, red line in Figure 1A). Qualitatively, the different optical properties of the $\mathrm{Au}-\mathrm{Fe}$ nanoalloys can be appreciated because its solution exhibits a brownish color, in contrast to the purple-red of the pure Au NPs (inset of Figure 1A). 

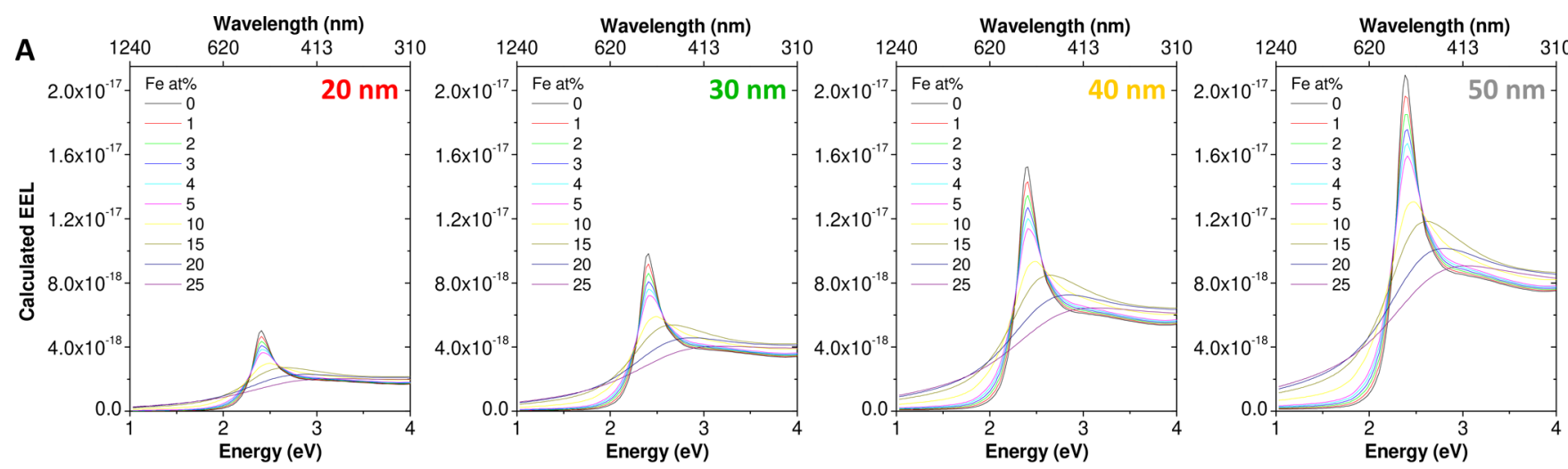

B

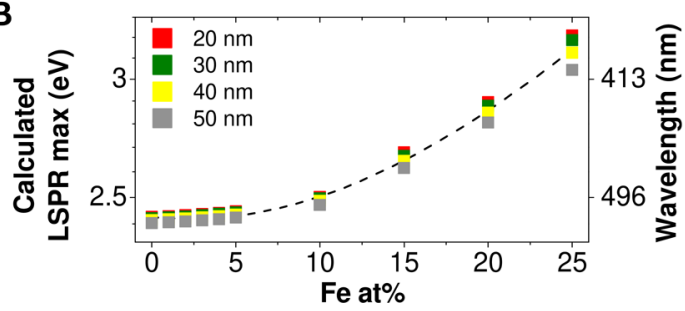

C

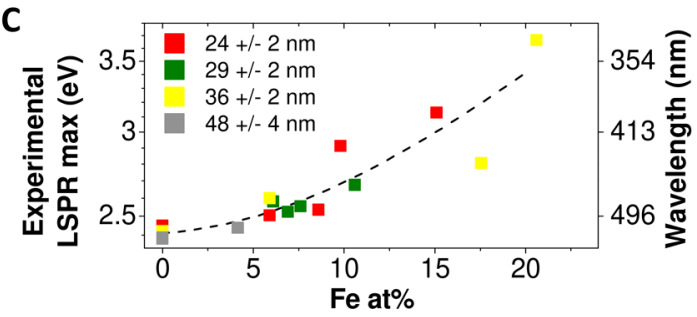

Figure 3. (A) Simulated EELS spectra showing the influence of the composition on the spectral properties of the alloy NP for various diameters. (B) Plot of LSPR maximum versus composition for the four sets of NPs considered in the calculations. (C) LSPR maxima extracted from the experimental spectra of Figure 2. The dashed black lines in (B) and (C) represent a second order polynomial fit of all the reported data (see Table S1 in SI for fitting parameters).

The Mie model provides a good description of the optical properties of spherical metal nanoparticles, when an appropriate optical constant is available. In this case, by providing as input data the size distribution measured from transmission electron microscopy (TEM) analysis (Figure $1 \mathrm{~B}$ and inset of Figure 1C, and setting the optical constant to that of an $\mathrm{Au}(88) \mathrm{Fe}(12)$ alloy according to the composition extracted from XRD data and from previous reports, ${ }^{19,29,30}$ we obtain a good agreement between the extinction spectrum calculated with the Mie model (black line in Figure 1C) and the experimental spectrum of the $\mathrm{Au}-\mathrm{Fe}$ nanoalloys (black line in Figure 1A). In particular, the spectrum calculated with the Mie model reproduces both the damping and position of the LSPR at $2.53 \mathrm{eV}(490 \mathrm{~nm})$, i.e., blue-shifted compared to that of $\mathrm{Au}$ NPs. Similarly, when the optical constant is set to that of pure $\mathrm{Au}$, a sharp LSPR peak appears at $2.38 \mathrm{eV}(520 \mathrm{~nm}$, red line in Figure $1 \mathrm{C}$ ), as in the reference experimental spectrum of the single element Au NPs (red line in Figure 1A). Note that these differences are due to the alloy composition and are not an artifact of NP size polydispersity, as proven in Figure S3 in the $\mathrm{SI}$, and in a previous study conducted with size-selected $\mathrm{Au}-$ Fe alloy nanoparticles. ${ }^{30}$

To deconvolve the contribution of the single $\mathrm{Au}-\mathrm{Fe}$ alloy NP to the extinction properties of their ensemble, using EELS we analyzed the LSPR features of isolated NPs from the same colloid deposited on a Si-N TEM grid. As apparent from Figure $1 \mathrm{C}$, the $\mathrm{Au}-\mathrm{Fe}$ sample has a wide size distribution, which allowed the analysis of $\mathrm{Au}-\mathrm{Fe}$ nanoalloy NPs with different compositions and diameters, while keeping the other experimental parameters unchanged. Then, in a step-by-step experimental approach, the plasmonic responses of these NPs were compared to those of pure Au NPs across similar size ranges. The NP dimensions were measured using the images acquired with the high angle annular dark-field (HAADF)STEM mode. For each $\mathrm{Au}-\mathrm{Fe} \mathrm{NP}$, we also performed EDXS analysis in order to measure its average composition and verify the homogeneity in the distribution of the iron within it. This potential for correlative analytics of nanoalloys is a strong point of EELS, when compared to other common spectroscopic methods for identifying single particle optical properties, such as dark field microscopy. The EDXS analysis confirms that $\mathrm{Au}$ and $\mathrm{Fe}$ are homogeneously distributed in all of the NPs considered, as expected for an alloy, and with no indications of oxidation (for example, see Figure 1D and Figure S4 in the SI). Besides, reanalysis of a colloid, dried on a Si-N TEM grid over two years earlier, by STEM-EDXS identified exactly the same characteristics of uniform alloy composition across individual, separated nanoparticles, indicating that the $\mathrm{Au}-\mathrm{Fe}$ nanoparticles are stable over time.

As shown in Figure 2A, the iron content of all the sampled $\mathrm{Au}-\mathrm{Fe}$ nanoalloy NPs spans between 4 and 20 at \%, with a volume weighted average over all the nanoparticles analyzed resulting in $10 \pm 4$ at $\%$. This agrees with our previous reports $^{29,30}$ and is close to the value used for the estimation done with Mie theory on the colloidal solution. Since the EELS measurements give direct insight into the near field distribution of the plasmon excitation, first we determined the EELS intensity map of the LSPR on a single $\mathrm{Au}-\mathrm{Fe} \mathrm{NP}$ with 9 at $\%$ of $\mathrm{Fe}$, finding that the highest intensity is located rather symmetrically along the edge of the nanoalloy (Figure 2B), in analogy to pure Au NPs. ${ }^{34}$ This behavior allows the analysis of the EELS response by measuring only a small portion of the NP along its edge, and then extracting the associated EELS spectrum to measure the LSPR peak position and shape. As explained in the Methods (see SI), this approach was applied to most of the measured NPs.

At the single particle level, the dependence of the LSPR on the composition was investigated on four groups of NPs with average sizes of $48 \pm 4,36 \pm 2,29 \pm 2$, and $24 \pm 2 \mathrm{~nm}$, respectively, identified by the red, yellow, green, and gray 
A

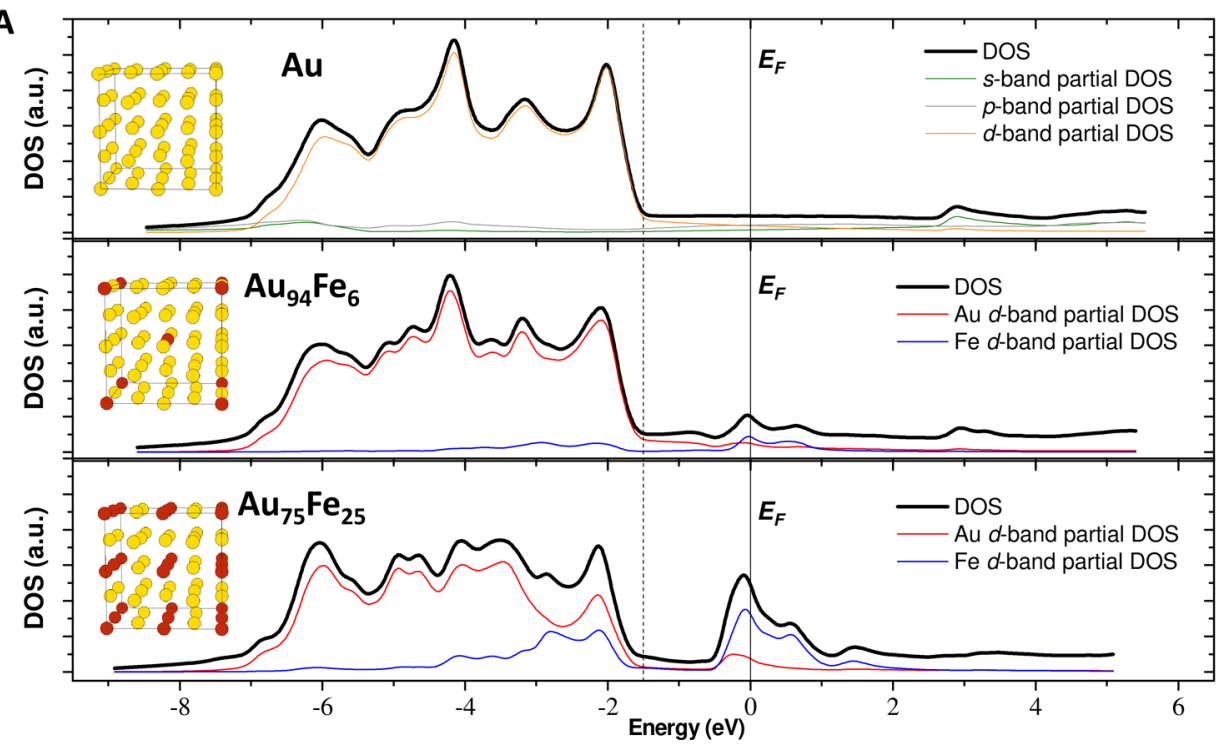

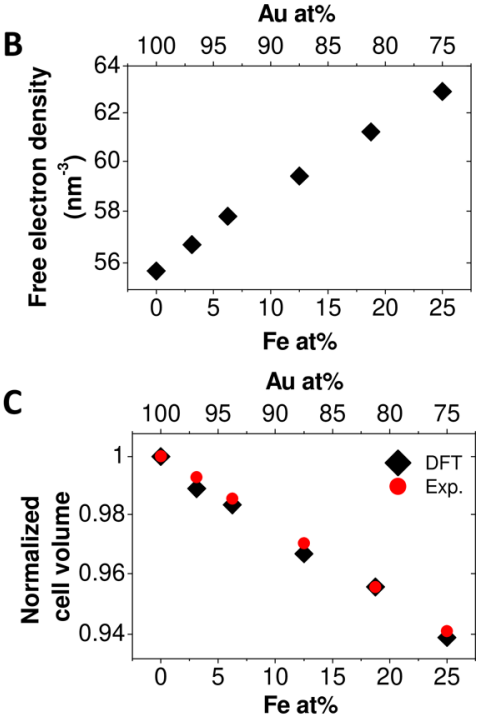

Figure 4. (A) DOS plot for $\mathrm{Au}$ and two representative $\mathrm{Au}-\mathrm{Fe}$ alloy compositions, showing the appearance of Fe $d$-band levels around the Fermi energy (indicated with a continuous vertical black line). In the Au-Fe alloys, the relative contributions from Au and Fe $d$-bands are plotted, respectively, with red and blue lines. The black dashed line located at the onset of the $\mathrm{Au} d$-band helps in highlighting the energy shift while increasing the Fe content. Insets show the models of cubic cells used in DFT calculations (Au atoms in yellow, Fe atoms in red). See SI for more details about Fermi energy calculation. (B) Free electron density versus Au-Fe alloy composition, from DFT calculations. (C) Plot of the normalized cell volume versus $\mathrm{Au}-\mathrm{Fe}$ alloy composition obtained from DFT (black diamonds) and experimental values extracted from ref 29 (red circles).

rectangles in Figure 2A. Previously, it was formally proved that EELS is related to the extinction (the sum of absorption and scattering) cross section in the case of small nanospheres. ${ }^{36}$ In fact, the EELS spectra collected on the Au-Fe NPs, reported in Figures $2 \mathrm{C}-\mathrm{G}$, all show an LSPR peak centered in the proximity of $2.5 \mathrm{eV}$ energy loss, superimposed on an absorption edge due to the single-electron interband transitions of the metal. ${ }^{37}$ However, the plasmonic peaks of these nanoalloys are found to vary in intensity and position depending on the dimensions and the fraction of $\mathrm{Fe}$. In particular, there is a general trend of a blue shift and damping of the LSPR as the fraction of $\mathrm{Fe}$ increases. This effect is especially evident when the $\mathrm{Au}-\mathrm{Fe}$ nanoalloys spectra are compared to those of pure Au NPs, as in Figure 2C,E,F.

Concerning the changes in LSPR with NP dimension, this is well described by considering the set of NPs identified by the blue rectangle in Figure 2A, with average iron content of $5.8 \pm$ 1.0 at $\%$ and size ranging from 21.0 to $46.5 \mathrm{~nm}$. From these spectra, reported in Figure $2 \mathrm{G}$, one can clearly observe that the LSPR becomes broader and less intense when the NP size decreases. This behavior follows that often observed in pure $\mathrm{Au}$ NPs and is attributed to intrinsic size effects. ${ }^{2,45,58}$ The intrinsic size effect acts on the electron relaxation frequency due to the confinement of conduction electrons in a volume with size comparable to their mean free path, and it becomes especially relevant when particle size is below $20 \mathrm{~nm}$. $^{2,45,58}$ However, since a plasmon peak is clearly identifiable in all spectra of Figure $2 \mathrm{G}$, the damping of LSPR due to the size effect is clearly less intense than that due to the increase of Fe content in the alloy.

To support the interpretation of the EELS measurements, a full wave analysis based on the SIE method was performed. The variables considered in the simulations were the diameter and the composition of the nanoalloys, assumed to have spherical shapes. From the simulated EELS spectra represented in Figure 3A, it is possible to observe that decreasing diameter does not significantly change the shape of the spectra but does lead to a decrease of the LSPR intensity. For instance, the EEL intensity decreases by a factor of $\sim 4$ going from 50 to $20 \mathrm{~nm}$ diameter nanoparticles. Since the optical response of a small metallic nanoparticle is dominated by absorption, one could expect a change in the extinction that follows the nanoparticle volume. However, the losses are proportional to the field inside the nanoparticle, which is neither homogeneous nor proportional to the size. For example, the ratio of the maximum of extinction for a plane-wave excitation of gold spheres of diameters 50 and $20 \mathrm{~nm}$ is only $\sim 3$. Additionally, the electron excitation is extremely localized, thus strongly affecting the field repartition inside the nanoparticles.

Conversely, the composition of the nanoparticles has a strong influence on both the position and the intensity of the plasmonic peak. A blue shift from 2.40 to $3.00 \mathrm{eV}$ and a damping of the plasmonic peak are observed as the $\mathrm{Fe}$ atomic fraction increases from 0 to 25 at $\%$. Figure $3 \mathrm{~B}, \mathrm{C}$ shows the comparison of the calculated and experimental position of the LSPR maximum, evaluated as the point where the derivative of the EELS spectrum is 0 versus NP composition. Although this method is not accurate when the LSPR is highly damped, as in the case of $\mathrm{Au}-\mathrm{Fe}$ nanoalloys with $\mathrm{Fe}$ content exceeding 10 at $\%$, the general trend clearly shows the damping and the blue shift of the plasmon peak by increasing the level of iron doping. The experimental blue shift follows a supralinear dependence on the iron atomic content, which is also found in the calculated EELS spectra (dashed lines in Figure 3B,C), and can be fitted with a second order polynomial (see Table S1 in SI for fitting parameters).

We note that a complete comparison between the simulations and the measurements is not trivial, partially due to the challenges of exactly removing the contributions of the zero-loss peak of elastically scattered electrons and the $\mathrm{Si}-\mathrm{N}$ 
membrane from the experimental LSPR EELS spectra (see Methods in SI for details on the data processing). However, all the general features of the measured spectra are reproduced by the simulations, including the peak broadening and blue shift as the $\mathrm{Fe}$ content increases.

In order to confirm further that LSPR blue shift and damping are due to alloy formation, numerical calculations using the discrete dipole approximation were performed for a series of $\mathrm{Au}$ nanospheres doped with an increasing volume fraction of either metal iron or iron oxide subnanometric clusters (see Figure S5A in SI). The results, shown in Figure S5B-E of the SI, clearly demonstrate that such doping does not generate the observed LSPR damping and blue shift.

Notably, the LSPR dependence on alloy composition, observed experimentally and theoretically, indicates that $\mathrm{Fe}$ has a deep effect on the electronic structure of $\mathrm{Au}$, producing a blue shift and a damping of the plasmon properties, which do not correspond to those given by the weighted average of the optical properties of the two pure metals. This is different from binary alloys of plasmonic metals, such as $\mathrm{Au}-\mathrm{Ag}$, which supports plasmons across all the composition range. ${ }^{25,59}$ It is known from literature that the introduction of Fe dopants in the Au lattice is associated with the appearance of iron $d$ states lying below the Fermi surface in the nanoalloy, from which new single electron transitions to the conduction band are possible. ${ }^{10,28,60}$ Low-frequency interband transitions are found in alloys of noble metals and transition metals with partially occupied $d$ states, ${ }^{10,61,62}$ which strongly affect the LSPR width because plasmon excitations can rapidly decay into electronhole pairs by Landau damping. ${ }^{63,64}$ The excitation of one electron in the conduction band and the creation of a hole in the lower-lying $d$ band has a remarkable contribution to the electron-electron relaxation rate of $\mathrm{Au}-\mathrm{Fe}$ nanoalloys ${ }^{10,61,62}$ and is responsible for the nonlinear dependence of the plasmon damping versus iron concentration observed experimentally. ${ }^{29,30}$ This mechanism is frequently found in nanoplasmonics, being at the origin of the superior plasmon performances of silver nanostructures in comparison with gold ones. $^{25,65,66}$ A further confirmation comes from DFT calculation of the density of states (DOS) in iron-doped $\mathrm{Au}$ crystals (Figure 4A), which shows the emergence of a new band around the Fermi level having a dominant Fe $d$ character. Concurrently, the edge of the $\mathrm{Au} d$-band slowly moves toward lower energies, with respect to the Fermi level. Hence, interband transitions are affected by the DOS changes in two ways: electronic excitations from the $\mathrm{Au} d$-bands shift toward higher energy as the $\mathrm{Fe}$ concentration rises, while the appearance of a new band close to the Fermi level makes possible the already mentioned low frequency electronic transitions, which greatly contribute to Landau damping.

Further to this, the experimental and computational results we present in Figure 3 show that the LSPR is blue-shifted with increasing iron content, following a supralinear dependence on the composition. The blue shift introduces an additional contribution to plasmon damping in $\mathrm{Au}-\mathrm{Fe}$ nanoalloys, due to the larger overlap with interband transitions inherent to the gold band structure. A blue shift of the LSPR is typically associated with an increase of the free electron density in the plasmonic metal, ${ }^{2}$ which in this case could be provoked by a contraction of the FCC lattice parameter of the gold lattice when alloyed with iron and by the replacement of $\mathrm{Au}$ with $\mathrm{Fe}^{29,30}$ DFT calculation confirms the increase in density of conduction electrons when passing from pure $\mathrm{Au}$ to the $\mathrm{Au}-$
Fe alloy (Figure 4B and Table S2 in SI). Two phenomena concur to this effect: ( $i$ ) The FCC lattice undergoes a contraction induced by the inclusion of $\mathrm{Fe}$ atoms (Figure $4 \mathrm{C}$ and Table S2 in SI), which is almost linear with $\mathrm{Fe}$ concentration and accounts for a small increase in the free electron density; (ii) $\mathrm{Fe}$ atoms push additional electron charge in the free electron density of the bimetallic crystal (Table S2 in SI), which in our model can be quantified as $\sim 1.3$ electrons per $\mathrm{Fe}$ atom in the alloy, whereas each $\mathrm{Au}$ atom contributes one free electron per atom.

It is worth mentioning that this model provides an almost linear increase of the free electron density with the amount of $\mathrm{Fe}$, whereas the parabolic variation of the LSPR maximum suggests a supralinear growth. However, the latter can be explained by the simultaneous energy shift of the $\mathrm{Au} d$-band levels and the appearance of new Fe $d$ levels in proximity of the Fermi energy, as observed from band structure and DOS calculations of the Au-Fe alloys. Specifically, simple considerations based on a Drude-Lorentz model ${ }^{67,68}$ for the alloy optical constant show that the LSPR frequency $\left(\omega_{L S P R}\right)$ generally follows a transcendental equation (see SI for details):

$$
\omega_{L S P R} \cong \frac{\omega_{p}^{*}}{\sqrt{\varepsilon_{\infty}+2 \varepsilon_{h}+\frac{f^{2}}{\Delta \Omega^{2}}}}
$$

where $\omega_{p}^{*}=\sqrt{\omega_{p}^{2}+\varphi^{2}}$ is the adjusted bulk plasma frequency, $\varphi$ the coupling strength associated with the low frequency interband transitions from $\mathrm{Fe} d$-states, $f$ the coupling strength associated with the interband transitions from the $\mathrm{Au}$ d-band, $\Delta \Omega^{2}=\omega_{1}^{2}-\omega_{L S P R}^{2}$ a detuning term measuring the distance between the squared frequency of the $\mathrm{Au} d$-band contribution and the squared LSPR frequency, $\varepsilon_{\infty}$ the contribution of higher energy electronic transitions to the metallic dielectric function, and $\varepsilon_{h}$ the host medium optical constant. On the one hand, the expression for $\omega_{p}^{*}$ indicates that there is an effective increase in plasma frequency, which is bound to the DOS of $\mathrm{Fe} d$-states. ${ }^{67,68} \mathrm{On}$ the other hand, from eq 1 , we see that $\omega_{L S P R}$ remains bounded from above by $\omega_{1}$ : $\Delta \Omega^{2}$ cannot become too small (or negative), as then the metal becomes absorptive and there is no plasmonic response. Importantly, the increase of the bulk plasma frequency alone does not guarantee a corresponding increase in the $\omega_{L S P R}$, if the detuning term $\Delta \Omega^{2}$ decreases. For instance, $\mathrm{Au}$ and $\mathrm{Ag}$ have almost identical bulk plasma frequencies of $8.6 \mathrm{eV},{ }^{68}$ but different $d$-band energies, around 2.7 and $4.2 \mathrm{eV}$, respectively, ${ }^{25,65}$ resulting in markedly different LSPR frequencies. ${ }^{2,10}$ Then, assuming that all other parameters remained fixed in eq 1 , an increase in $\omega_{L S P R}$ must come from the combined effect of an increase in the adjusted bulk plasma frequency $\omega_{p}^{*}$ and an increase in frequency of the $\mathrm{Au} d$-band contribution $\omega_{1}$. Although the Drude-Lorentz model is not accurate enough to reproduce the experimental results at the level of SIE and Mie theory, both trends are observed in our experiments and theory, therefore justifying the measured supralinear increase of the LSPR frequency with Fe content.

Regarding plasmon broadening, we find that the electron relaxation rate $\gamma_{0}^{*}$ also gets augmented by the introduction of the shallow Fe $d$-states (see SI for details):

$$
\gamma_{0}^{*}=\gamma_{0} \frac{\omega_{p}{ }^{2}}{\omega_{p}^{2}+\varphi^{2}}+\gamma_{2} \frac{\varphi^{2}}{\omega_{p}^{2}+\varphi^{2}}
$$


where $\gamma_{0}$ is the free electron relaxation rate and $\gamma_{2}$ is the relaxation rate of the shallow $\mathrm{Fe} d$-states. This is essentially a manifestation of the Matthiessen rule, ${ }^{68}$ where the total plasma relaxation rate is the weighted sum of two rates. The observed large LSPR broadening with Fe content points to $\gamma_{2} \gg \gamma_{0}$ and thus to the fact that the added $\mathrm{Fe} d$-electrons introduce relaxation (i.e., new relaxation pathways and thus broadening) into an otherwise little damped Au electron gas.

In summary, we have introduced a strategy for predicting and interpreting the optical properties of nanoalloys combining EELS measurements and DFT calculations, here applied to the investigation of the LSPR in $\mathrm{Au}-\mathrm{Fe}$ alloy NPs with different compositions and sizes. We found that the LSPR is present in $\mathrm{Au}-\mathrm{Fe}$ nanoalloys, although with an intensity and spectral position dependent on the structural parameters of the NP. In particular, we observed that, as the fraction of Fe increases, the plasmonic peak is damped and blue-shifted following a supralinear dependence on the Fe atomic content. By relating the experimental findings to EELS simulations, we verified that we can predict the plasmonic properties of nanoalloys with different dimensions and compositions. Changes in the plasmonic properties of the alloy are due to the increase of the conduction electron density, the emergence of new states around the Fermi level, and the energy shift of the Au $d$ band, as deduced from DFT calculations and use of the DrudeLorentz model. Although the Fe doping induces a damping of the LSPR, additional Mie theory calculations show that the extinction, scattering, and absorption cross sections remain in the same order of magnitude (Figure S6 in SI). Further to this, numerical calculations show that the local field enhancement in $\mathrm{Au}-\mathrm{Fe}$ nanoalloys is still appreciable and comparable to that of pure Au NPs of the same size (see Figure S7 in SI). Since Au$\mathrm{Fe}$ alloys have a spin-glass behavior below 16 at \% Fe, reentrant spin-glass state from $16-24$ at $\% \mathrm{Fe}$, and fully ferromagnetic state above 24 at $\% \mathrm{Fe}^{29}$ these nanoalloys are therefore very promising for the investigation of hybrid magnetic-plasmonic effects ${ }^{14,15,53}$ and several applications exploiting the multimodal magnetic-plasmonic features in sensing ${ }^{11}$ and nanomedicine. ${ }^{19}$

Overall, this study provides direct insights into the plasmonics of nanoalloys made of noble metals and transition metals, pointing to alloying as a powerful strategy for modifying plasmonic performances through band structure engineering. By relating the plasmon modes and plasmoninterband interaction with the composition, this work also facilitates the development of hybrid plasmonic structures with tailored responses, beyond the possibilities offered by singleelement NPs. Due to the multiple functionalities of $\mathrm{Au}-\mathrm{Fe}$ and the other plasmonic nanoalloys, we expect that this study will provide new opportunities for the control of the nanoscale optical response, enabling efficient tuning of directional scattering, molecular sensing, thermo-plasmonics, bioimaging, photocatalysis, and local field enhancement for the amplification of spectroscopic signals.

\section{ASSOCIATED CONTENT}

\section{S Supporting Information}

The Supporting Information is available free of charge on the ACS Publications website at DOI: 10.1021/acs.nanolett.9b02396.

Experimental and computational methods, parameters of polynomial fits, table of DFT numerical results, XRD,
XPS, EDXS, Mie theory and DDA calculations, cell models for DFT calculations and additional DFT data, Drude-Lorentz model for $\omega_{L S P R}$ (PDF)

\section{AUTHOR INFORMATION}

\section{Corresponding Authors}

*E-mail: duncan.alexander@epfl.ch.

*E-mail: vincenzo.amendola@unipd.it.

ORCID

Duncan T. L. Alexander: 0000-0003-4350-8587

Elefterios Lidorikis: 0000-0002-9552-9366

Stefano Agnoli: 0000-0001-5204-5460

Gabriel D. Bernasconi: 0000-0001-7183-9178

Jérémy Butet: 0000-0001-9598-9074

Olivier J. F. Martin: 0000-0002-9574-3119

Vincenzo Amendola: 0000-0002-9937-7005

Notes

The authors declare no competing financial interest.

\section{ACKNOWLEDGMENTS}

This work was performed in the context of the European COST Action MP1302 Nanospectroscopy. V.A. acknowledges the support by the University of Padova STARS grant "4NANOMED" and the Italian Ministry of Foreign Affairs and International Cooperation (General Directorate for the Promotion of the Country System) "Great relevance project" with protocol number 0191594. We acknowledge the CINECA award under the ISCRA initiative, for the availability of high performance computing resources and support. Funding from the European Research Council (ERC-2015AdG-695206 Nanofactory) and the Swiss National Science Foundation (project 200020_153662) is gratefully acknowledged. The Interdisciplinary Centre for Electron Microscopy (CIME) at EPFL is thanked for access to its facilities.

\section{REFERENCES}

(1) Maier, S. A. Plasmonics: Fundamentals and Applications; Springer, 2007.

(2) Amendola, V.; Pilot, R.; Frasconi, M.; Maragò, O. M.; Iatì, M. A. J. Phys.: Condens. Matter 2017, 29, 203002.

(3) Lal, S.; Link, S.; Halas, N. J. Nat. Photonics 2007, 1, 641-648.

(4) Ben-Shahar, Y.; Scotognella, F.; Kriegel, I.; Moretti, L.; Cerullo, G.; Rabani, E.; Banin, U. Nat. Commun. 2016, 7, 10413.

(5) Halas, N. J.; Lal, S.; Chang, W.-S.; Link, S.; Nordlander, P. Chem. Rev. 2011, 111, 3913-3961.

(6) Xia, Y.; Halas, N. J. MRS Bull. 2005, 30, 338-348.

(7) Haes, A. J.; Haynes, C. L.; McFarland, A. D.; Schatz, G. C.; Van Duyne, R. P.; Zou, S. MRS Bull. 2005, 30, 368-375.

(8) Cortie, M. B.; McDonagh, A. M. Chem. Rev. 2011, 111, 37133735 .

(9) Naik, G. V.; Shalaev, V. M.; Boltasseva, A. Adv. Mater. 2013, 25, 3264-3294.

(10) Blaber, M. G.; Arnold, M. D.; Ford, M. J. J. Phys.: Condens. Matter 2010, 22, 143201.

(11) Peng, S.; Lei, C.; Ren, Y.; Cook, R. E.; Sun, Y. Angew. Chem., Int. Ed. 2011, 50, 3158-3163.

(12) Wang, D.; Li, Y. Adv. Mater. 2011, 23, 1044-1060.

(13) Cable, R. E.; Schaak, R. E. Chem. Mater. 2007, 19, 4098-4104.

(14) Bogani, L.; Cavigli, L.; de Julián Fernández, C.; Mazzoldi, P.; Mattei, G.; Gurioli, M.; Dressel, M.; Gatteschi, D. Adv. Mater. 2010, 22, 4054-4058.

(15) Armelles, G.; Cebollada, A.; García-Martín, A.; González, M. U. Adv. Opt. Mater. 2013, 1, 10-35. 
(16) Martirez, J. M. P.; Carter, E. A. J. Am. Chem. Soc. 2017, 139, 4390-4398.

(17) Martirez, J. M. P.; Carter, E. A. ACS Nano 2016, 10, 29402949.

(18) Quiroz, J.; Barbosa, E. C. M.; Araujo, T. P.; Fiorio, J. L.; Wang, Y.-C.; Zou, Y.-C.; Mou, T.; Alves, T. V.; de Oliveira, D. C.; Wang, B.; et al. Nano Lett. 2018, 18, 7289-7297.

(19) Amendola, V.; Scaramuzza, S.; Litti, L.; Meneghetti, M.; Zuccolotto, G.; Rosato, A.; Nicolato, E.; Marzola, P.; Fracasso, G.; Anselmi, C.; et al. Small 2014, 10, 2476-2486.

(20) Scaramuzza, S.; Badocco, D.; Pastore, P.; Coral, D. F.; Fernández van Raap, M. B.; Amendola, V. ChemPhysChem 2017, 18, 1026-1034.

(21) Amendola, V.; Scaramuzza, S.; Agnoli, S.; Granozzi, G.; Meneghetti, M.; Campo, G.; Bonanni, V.; Pineider, F.; Sangregorio, C.; Ghigna, P.; et al. Nano Res. 2015, 8, 4007-4023.

(22) Amendola, V.; Sajia, R.; Maragò, O. M. M.; Iatì, A.; Saija, R.; Maragò, O. M. M.; Iatì, M. A.; Sajia, R.; Maragò, O. M. M.; Iatì, A. Nanoscale 2015, 19, 8782-8792.

(23) Vassalini, I.; Borgese, L.; Mariz, M.; Polizzi, S.; Aquilanti, G.; Ghigna, P.; Sartorel, A.; Amendola, V.; Alessandri, I. Angew. Chem., Int. Ed. 2017, 56, 6589-6593.

(24) Novotny, L.; van Hulst, N. Nat. Photonics 2011, 5, 83-90.

(25) Gong, C.; Kaplan, A.; Benson, Z. A.; Baker, D. R.; McClure, J. P.; Rocha, A. R.; Leite, M. S. Adv. Opt. Mater. 2018, 6, 1-7.

(26) Ferrando, R.; Jellinek, J.; Johnston, R. L. Chem. Rev. 2008, 108, $845-910$.

(27) Okamoto, H.; Massalski, T. B.; Swartzendruber, L. J.; Beck, P. A. Bull. Alloy Phase Diagrams 1984, 5, 592-601.

(28) Lee, Y. P.; Kudryavtsev, Y. V.; Nemoshkalenko, V. V.; Gontarz, R.; Rhee, J. Y. Phys. Rev. B: Condens. Matter Mater. Phys. 2003, 67, 104424.

(29) Amendola, V.; Meneghetti, M.; Bakr, O. M.; Riello, P.; Polizzi, S.; Fiameni, S.; Dalaver, H.; Arosio, P.; Orlando, T.; de Julian Fernandez, C.; et al. Nanoscale 2013, 5, 5611-5619.

(30) Amendola, V.; Scaramuzza, S.; Agnoli, S.; Polizzi, S.; Meneghetti, M. Nanoscale 2014, 6, 1423-1433.

(31) Scaramuzza, S.; Agnoli, S.; Amendola, V. Phys. Chem. Chem. Phys. 2015, 17, 28076-28087.

(32) Amendola, V.; Scaramuzza, S.; Carraro, F.; Cattaruzza, E. J. Colloid Interface Sci. 2017, 489, 18-27.

(33) Flauraud, V.; Bernasconi, G. D.; Butet, J.; Mastrangeli, M.; Alexander, D. T. L.; Martin, O. J. F.; Brugger, J. ACS Photonics 2017, 4, 1661-1668.

(34) Flauraud, V.; Bernasconi, G. D.; Butet, J.; Alexander, D. T. L.; Martin, O. J. F.; Brugger, J. ACS Nano 2017, 11, 3485-3495.

(35) Bernasconi, G. D.; Butet, J. my; Flauraud, V.; Alexander, D.; Brugger, J.; Martin, O. J. F. ACS Photonics 2017, 4, 156-164.

(36) García de Abajo, F. J. Rev. Mod. Phys. 2010, 82, 209-275.

(37) Wu, Y.; Li, G.; Camden, J. P. Chem. Rev. 2018, 118, 29943031.

(38) Lourenço-Martins, H.; Das, P.; Tizei, L. H. G.; Weil, R.; Kociak, M. Nat. Phys. 2018, 14, 360-364.

(39) Barrow, S. J.; Collins, S. M.; Rossouw, D.; Funston, A. M.; Botton, G. A.; Midgley, P. A.; Mulvaney, P. ACS Nano 2016, 10, $8552-8563$.

(40) Barrow, S. J.; Rossouw, D.; Funston, A. M.; Botton, G. A.; Mulvaney, P. Nano Lett. 2014, 14, 3799-3808.

(41) Byers, C. P.; Hoener, B. S.; Chang, W.-S.; Yorulmaz, M.; Link, S.; Landes, C. F. J. Phys. Chem. B 2014, 118, 14047-14055.

(42) Haberfehlner, G.; Schmidt, F.-P.; Schaffernak, G.; Hörl, A.; Trügler, A.; Hohenau, A.; Hofer, F.; Krenn, J. R.; Hohenester, U.; Kothleitner, G. Nano Lett. 2017, 17, 6773-6777.

(43) Schmidt, F. P.; Ditlbacher, H.; Hofer, F.; Krenn, J. R.; Hohenester, U. Nano Lett. 2014, 14, 4810-4815.

(44) Schmidt, F.-P.; Ditlbacher, H.; Hohenau, A.; Hohenester, U.; Hofer, F.; Krenn, J. R. Nano Lett. 2016, 16, 5152-5155.
(45) Bosman, M.; Ye, E.; Tan, S. F.; Nijhuis, C. A.; Yang, J. K. W.; Marty, R.; Mlayah, A.; Arbouet, A.; Girard, C.; Han, M.-Y. Sci. Rep. 2013, 3, 1312.

(46) Losquin, A.; Zagonel, L. F.; Myroshnychenko, V.; RodríguezGonzález, B.; Tencé, M.; Scarabelli, L.; Förstner, J.; Liz-Marzán, L. M.; de Abajo, F. J.; Stéphan, O. Nano Lett. 2015, 15, 1229-1237.

(47) Jain, P. K.; Xiao, Y.; Walsworth, R.; Cohen, A. E. Nano Lett. 2009, 9, 1644-1650.

(48) Jung, I.; Ih, S.; Yoo, H.; Hong, S.; Park, S. Nano Lett. 2018, 18, 1984-1992.

(49) Rollinger, M.; Thielen, P.; Melander, E.; Östman, E.; Kapaklis, V.; Obry, B.; Cinchetti, M.; García-Martín, A.; Aeschlimann, M.; Papaioannou, E. T. Nano Lett. 2016, 16, 2432-2438.

(50) Maccaferri, N.; Bergamini, L.; Pancaldi, M.; Schmidt, M. K.; Kataja, M.; van Dijken, S.; Zabala, N.; Aizpurua, J.; Vavassori, P. Nano Lett. 2016, 16, 2533-2542.

(51) Wang, L.; Clavero, C.; Huba, Z.; Carroll, K. J.; Carpenter, E. E.; Gu, D.; Lukaszew, R. A. Nano Lett. 2011, 11, 1237-1240.

(52) Geryak, R.; Geldmeier, J.; Wallace, K.; Tsukruk, V. V. Nano Lett. 2015, 15, 2679-2684.

(53) Maccaferri, N.; Gregorczyk, K. E.; de Oliveira, T. V. A. G.; Kataja, M.; van Dijken, S.; Pirzadeh, Z.; Dmitriev, A.; Åkerman, J.; Knez, M.; Vavassori, P. Nat. Commun. 2015, 6, 6150.

(54) Shegai, T.; Chen, S.; Miljković, V. D.; Zengin, G.; Johansson, P.; Käll, M. A. Nat. Commun. 2011, 2, 481.

(55) Mayer, K. M.; Hafner, J. H. Chem. Rev. 2011, 111, 3828-3857.

(56) Wadell, C.; Nugroho, F. A. A.; Lidström, E.; Iandolo, B.; Wagner, J. B.; Langhammer, C. Nano Lett. 2015, 15, 3563-3570.

(57) Zhang, X.; Chen, Y. L.; Liu, R.-S.; Tsai, D. P. Rep. Prog. Phys. 2013, 76, No. 046401.

(58) Scholl, J. A.; Koh, A. L.; Dionne, J. A. Nature 2012, 483, 421427.

(59) Bai, T.; Lu, P.; Zhang, K.; Zhou, P.; Liu, Y.; Guo, Z.; Lu, X. J. Biomed. Nanotechnol. 2017, 13, 1178-1209.

(60) Beaglehole, D.; Hendrickson, T. J. Phys. Rev. Lett. 1969, 22, $133-136$.

(61) Koike, H.; Yamaguchi, S.; Hanyu, T. J. Phys. Soc. Jpn. 1976, 40, 219-225.

(62) Hunderi, O. Solid State Commun. 1972, 11, 885-888.

(63) Brongersma, M. L.; Halas, N. J.; Nordlander, P. Nat. Nanotechnol. 2015, 10, 25-34.

(64) Li, X.; Xiao, D.; Zhang, Z. New J. Phys. 2013, 15, 23011.

(65) Arnold, M. D.; Blaber, M. G. Opt. Express 2009, 17, 38353847.

(66) Lalisse, A.; Tessier, G.; Plain, J.; Baffou, G. J. Phys. Chem. C 2015, 119, 25518-25528.

(67) Kreibig, U.; Vollmer, M. Optical Properties of Metal Clusters; Springer: Berlin, 1995.

(68) Ashcroft, N. W.; Mermin, N. D. Solid State Physics; Holt, Rinehart and Winston: New York, 1976. 